\title{
Dronabinol has preferential antileukemic activity in acute lymphoblastic and myeloid leukemia with lymphoid differentiation patterns
}

Kerstin Maria Kampa-Schittenhelm, Olaf Salitzky, Figen Akmut, Barbara Illing, Lothar Kanz, Helmut Rainer Salih and Marcus Matthias Schittenhelm*

\begin{abstract}
Background: It has been previously demonstrated in several cancer models, that Dronabinol (THC) may have anti-tumor activity - however, controversial data exists for acute leukemia. We have anecdotal evidence that THC may have contributed to disease control in a patient with acute undifferentiated leukemia.

Methods: To test this hypothesis, we evaluated the antileukemic efficacy of THC in several leukemia cell lines and native leukemia blasts cultured ex vivo. Expression analysis for the CB1/2 receptors was performed by Western immunoblotting and flow cytometry. CB-receptor antagonists as well as a CRISPR double nickase knockdown approach were used to evaluate for receptor specificity of the observed proapoptotic effects.

Results: Meaningful antiproliferative as well as proapoptotic effects were demonstrated in a subset of cases - with a preference of leukemia cells from the lymphatic lineage or acute myeloid leukemia cells expressing lymphatic markers. Induction of apoptosis was mediated via CB1 as well as CB2, and expression of CB receptors was a prerequisite for therapy response in our models. Importantly, we demonstrate that antileukemic concentrations are achievable in vivo.
\end{abstract}

Conclusion: Our study provides rigorous data to support clinical evaluation of THC as a low-toxic therapy option in a well defined subset of acute leukemia patients.

Keywords: Delta9-Tetrahydrocannabinol, Dronabinol, THC, Leukemia, AML, ALL

\section{Background}

Delta9-Tetrahydrocannabinol is the major psychoactive constituent of Cannabis sativa and signals through Gprotein-coupled cannabinoid receptors (CB).

The CB1 receptor is predominantly abundant in brain tissues [1]. In contrast, the CB2 receptor was initially described in the lymphatic system [2], but is also expressed in other tissues such as brain [3], brain endothelium [4], bone [5] or skin [6].

* Correspondence: marcus.schittenhelm@med.uni-tuebingen.de University Hospital Tübingen, Dept. of Oncology, Hematology, Rheumatology, Immunology and Pulmology, Tübingen, Germany
While the central CB1 receptor accounts for the psychotropic, analgetic, and orectic effects, the dominantly peripheral CB2 receptor is linked to immunomodulation [7] and regulation of bone mass [5] among other functions.

Despite the broadly acknowledged potential of cannabinoid agonists with regard to effective relief of tumor or neuropathic pain, muscular spasm or nausea-combined with an excellent safety profile and moderate sideeffects-clinical use is very restricted in most countries due to the unwanted psychoactive effects (reviewed by Pertwee [8]). The natural (-)- $\Delta^{9}$-Tetrahydrocannabinol isomer dronabinol (further referred to as THC) is a potent pan-cannabinoid receptor (CB1/2) agonist, which gained FDA-approval in the United States as $\mathrm{Marinol}^{\circ}$ for the 
treatment of chemotherapy-induced nausea and vomiting or stimulation of appetite in AIDS patients.

Moreover and importantly, there is evidence for growth-inhibiting effects in tumor models, including animal models, arguing for the use of cannabinoids as lowtoxic anticancer therapeutics (reviewed by Guzman [9]).

Anecdotal evidence has lead us to speculate that THC may have contributed to disease control in a patient with acute undifferentiated leukemia. Indeed, previous reports suggest a proapoptotic antitumor effect of $\mathrm{CB}$-agonists on acute leukemia cells in vitro [10-12]. These studies concentrate on the analysis of the Jurkat T-lymphoblastic cell line (which was established from the peripheral blood of a patient suffering from acute T-lymphoblastic leukemia in 1976; see also http//:https://www.dsmz.de). However, the mechanism of action is controversially discussed in these studies (CB1 versus CB2 mediation) [11, 12].

Even more controversially, other studies suggest a hematopoietic growth advantage mediated via CB2 activation - and utmost challenging, characterize CB2 as an oncoprotein linked to (myeloid) leukemogenesis [13-15].

We now provide data demonstrating potent antileukemic efficacy of THC in acute leukemia cell lines in vitro as well as freshly harvested native leukemia blasts cultured ex vivo. Notably, antiproliferative as well as proapoptotic effects are preferentially seen in leukemia cells of the lymphatic lineage or in acute myeloid leukemia cells expressing lymphatic markers.

\section{Results}

\section{THC inhibits cellular proliferation in lymphatic and} myeloid leukemia cell lines

In analogy to previous reports, we used the Tlymphoblastic leukemia cell line Jurkat to reconfirm whether THC is capable to inhibit cellular proliferation in an acute leukemia cell model. THC was administered for $72 \mathrm{~h}$ in a dose dependent manner and the antiproliferative effect, measured as the reduction of XTT metabolism in correlation to an untreated negative control, was measured accordingly. THC produced significant and dose-dependent inhibition of cellular proliferation (Fig. 1a) with a computed $\mathrm{IC}_{50} \sim 15 \mu \mathrm{M}$ in a non-linear regression analysis (Fig. 1b).

To determine, whether the observed effects are unique to the Jurkat cell line, we also tested an acute myeloid leukemia cell line, MOLM13 - and found similar antiproliferative effects with an $\mathrm{IC}_{50} \sim 18 \mu \mathrm{M}$ (Fig. 1c, d).

At the higher tested doses $>50 \mu \mathrm{M}$, virtual no metabolic activity was observed for both Jurkat as well as MOLM13 cells - arguing that cells are not viable and may have been directed to programmed cell death. In this context, it has been previously described, that cannabinoid agonists are capable to induce apoptosis in tumor cells [16].

\section{THC induces apoptosis in leukemia cell lines}

We addressed this question in an annexin V-based flow cytometry assay and treated Jurkat as well as MOLM13 cells with increasing concentrations of THC for $48 \mathrm{~h}$.

For Jurkats, we were able to demonstrate dosedependent induction of apoptosis with significant $\mathrm{p}$ values starting at $40 \mu \mathrm{M}$ in a Student's $t$-test (Fig. 2a). IC $\mathrm{I}_{50}$ was computed in a non-linear regression analysis at $\sim 46 \mu \mathrm{M}$ (Fig. 2b). No signs of cell cycle arrest with abrogation of the proapoptotic effect in higher doses [17] were seen: At the highest tested dose, $75 \mu \mathrm{M}$, a virtual complete kill of the entire population was observed (Fig. 2c).

Additional annexin V-staining data is provided in Additional file 1: Figure S1, demonstrating dose-dependent induction of early apoptosis in Jurkat cells treated with THC for $10 \mathrm{~h}$.

It has been previously demonstrated for Jurkat leukemia cells, that THC-mediated induction of apoptosis is linked to the intrinsic, mitochondrial pathway [10]. We confirmed this finding in Western immunoblots showing cleavage of caspases 3 and 9 upon treatment with THC (Fig. 5c and Additional file 2: Figure S2). Cleaved caspase 9 is known as a central mediator of the intrinsic mitochondrial apoptosis pathway.

Similarly, MOLM13 cells underwent induction of apoptosis in response to $\mathrm{THC}$ with a computed $\mathrm{IC}_{50} \sim$ $38 \mu \mathrm{M}$ and complete kill of cells at $75 \mu \mathrm{M}$ (Fig. $2 \mathrm{~d}-\mathrm{f}$ ).

A drug-carrier (i.e. methanol) control assay did not reveal any significant proapoptotic effects at the highest concentration used with the THC-dilution experiments.

To diminish individual cell line-specific effects, we expanded our analysis to other leukemia cell line models. For our experiments, we used MOLM14 cells, a sister cell line of MOLM13 derived from the same patient, as well as independent acute myeloid leukemia cell lines (MV4-11, M0-7e, HL60), the core binding factor cell line Kasumi1 and the acute blast crisis CML cell line K562.

All cell lines were treated with THC in a dose dependent manner and induction of apoptosis was measured after 24 and $48 \mathrm{~h}$.

Together, THC was capable of inducing apoptosis in all leukemia cell lines-whereas $\mathrm{IC}_{50 \mathrm{~s}}$ differed in between the tested cell lines. A summary of computed $\mathrm{IC}_{50 \mathrm{~s}}$ is provided with Table 1. Dose-effect plots and doseregression analysis for each cell line are provided as supplemental data (Additional file 3: Figure S3, Additional file 4: Figure S4, Additional file 5: Figure S5, Additional file 6: Figure S6, Additional file 7: Figure S7, Additional file 8: Figure S8).

\section{THC reduces the proportion of viable cells cultured ex vivo}

We next tested native leukemia blasts cultured ex vivo, with regard to the antileukemic sensitivity after exposure 


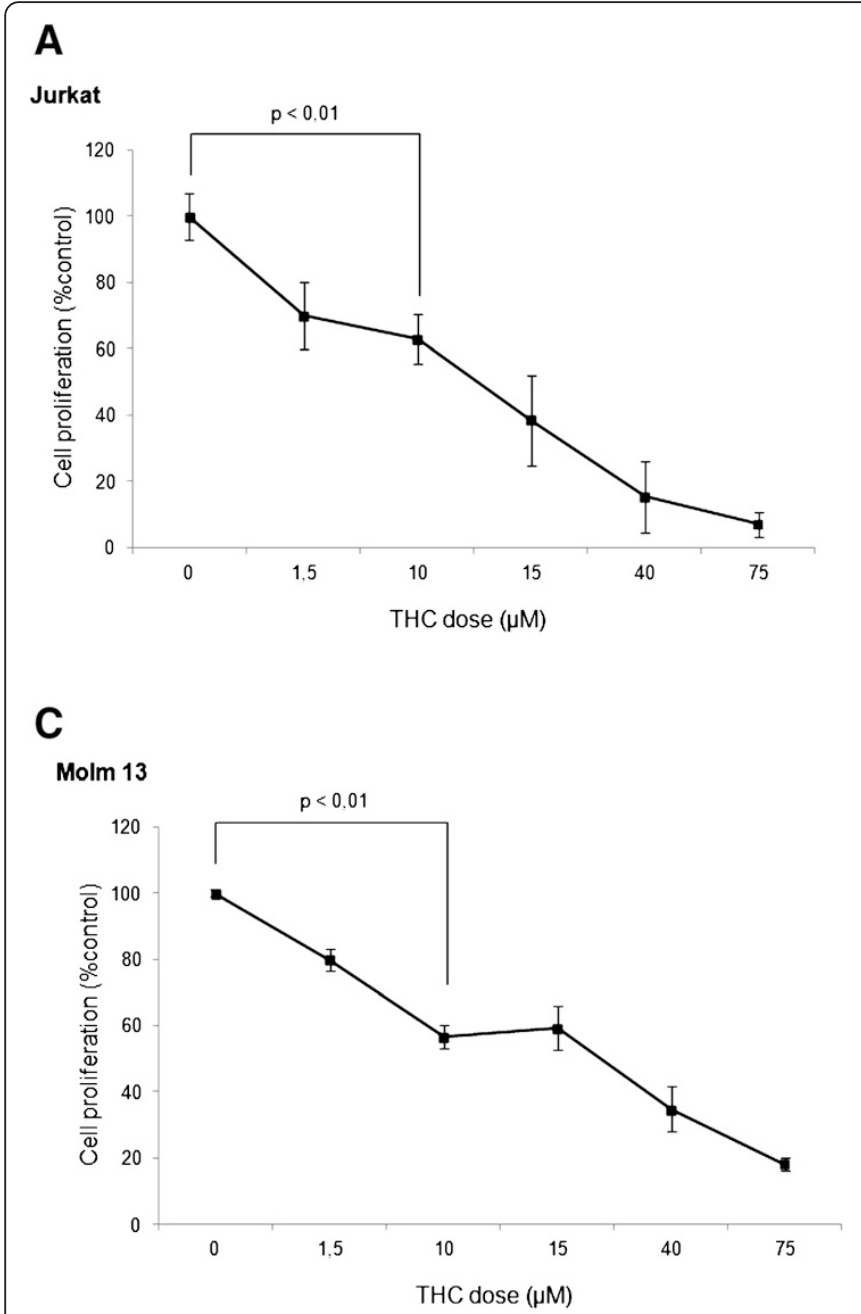

B

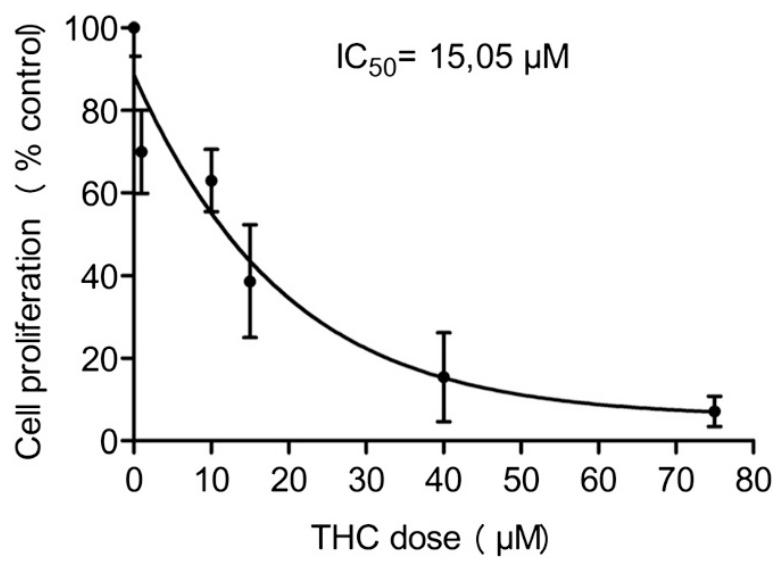

D

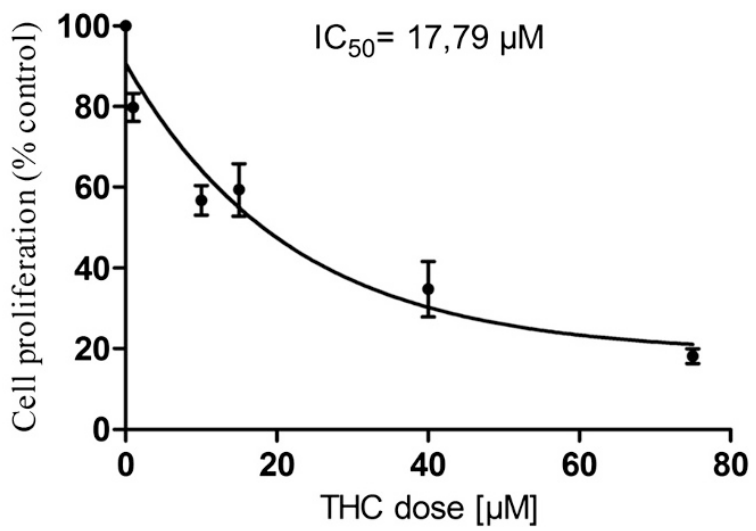

Fig. 1 Photometric XTT-analysis assaying metabolic active cells in dependence of THC concentration. Representative dose-effect curves for Jurkat (a) and MOLM13 (c) cells treated with THC in a dose-dependent manner are shown on. Student's t-test analysis reveals significant reduction of proliferating cells as indicated for two doses (statistical significance at $p<0.05$ ). Experiments were performed in triplicates. Linear regression analysis was performed to compute $I C_{50 s}$ for both cell lines (b and $\mathbf{d}$ )

to $\mathrm{THC}$ with doses in the range of $\mathrm{IC}_{50 \mathrm{~s}}$ for the Jurkat cell line.

As a relatively high basal proportion of dead/apoptotic cells was present in the freshly harvested and cultured cells, which is a commonly observed problem in ex vivo cell cultures, we used a flow cytometry-based assay as recently established by our group (Kampa-Schittenhelm et al. [18]) measuring reduction of the viable cell proportion in a FSC/SSC scatter plot. To ensure that the gated population is viable an annexin V/PI-based assay was performed simultaneously using THC-naïve cells. The viable cell fraction was defined as absence of annexin $\mathrm{V}$ or PI positivity and the gate was set accordingly. Further, immunophenotyping was set up to confirm the leukemic character of the gated population (i.e. CD45low+/-CD34 positivity). Reduction of the viable cell fraction was measured $48 \mathrm{~h}$ after THC exposure compared to treatment- naive parental cells. Density dot plots of a representative patient sample are provided in Fig. 3a-e.

Dose-effect waterfall bar graphs demonstrating reduction of viable cells in lymphatic as well as myeloid leukemia patient samples are provided with Fig. 3f (myeloid leukemia) and Fig. 3g (lymphatic leukemia). In general, leukemias with lymphatic differentiation were more sensitive to THC-with 9/13 (69 \%) patients showing an at least 50\% reduction of viable cells at $50 \mu \mathrm{M}$. In contrast, only $4 / 13$ (31 \%) patients with AML demonstrated a $\geq 50 \%$ reduction of the viable cell proportion.

\section{Response to THC correlates with expression of CB1 and CB2 receptors}

To evaluate, whether response to THC correlates with cannabinoid receptor expression, we measured protein expression levels of CB1 and CB2 on all available patient samples 

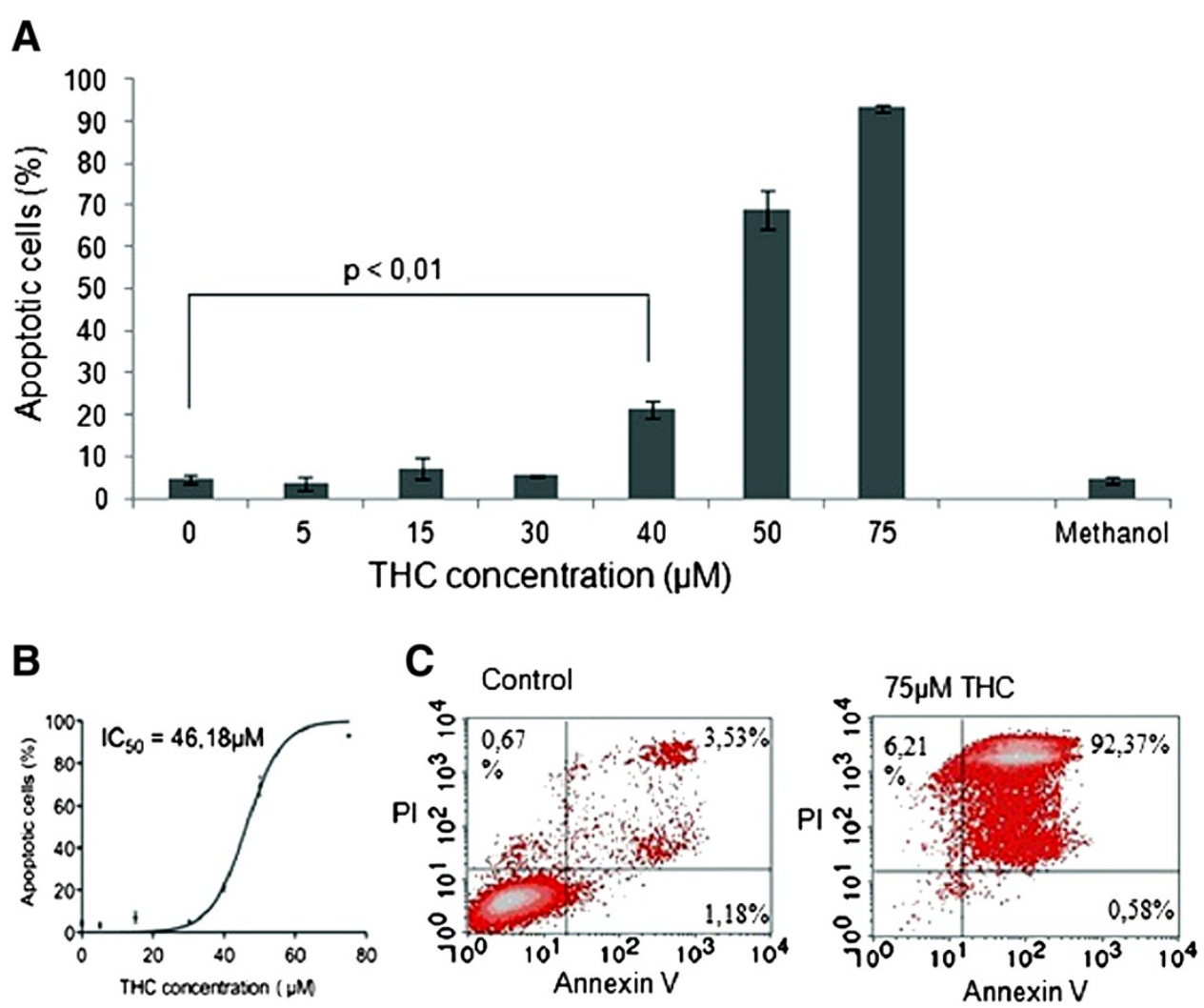

D
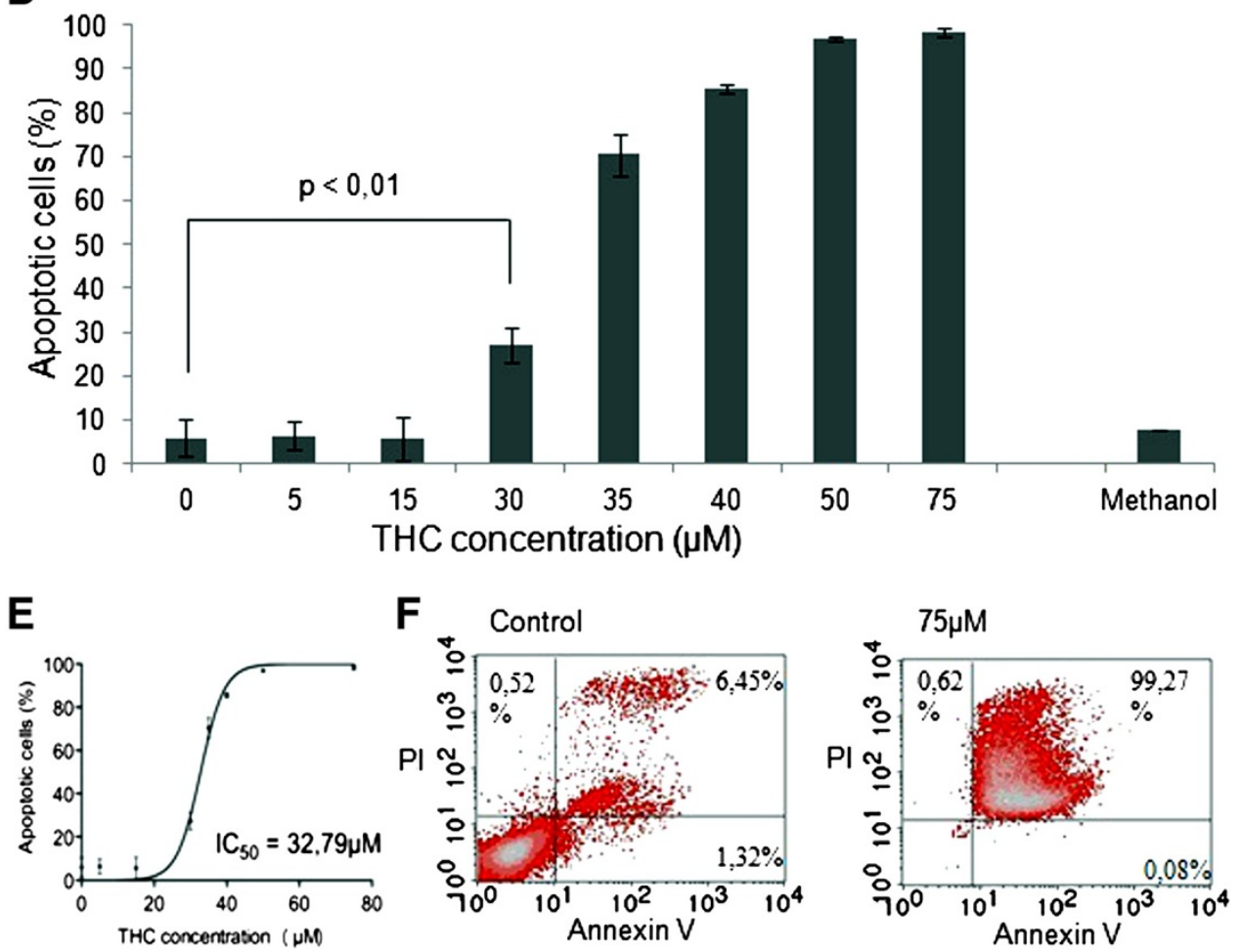

Fig. 2 (See legend on next page.) 
(See figure on previous page.)

Fig. 2 Flow cytometric apoptosis assay measuring early apoptotic (annexin $V$ ) and later phase apoptotic cells (propidium iodide) after exposure of Jurkat (a-c) or MOLM13 (d-f) cells to THC. Dose-effect curves for Jurkat (a) and MOLM13 (d) cells treated with THC in a dose-dependent manner are shown. Student's $t$-test demonstrates significance $(p<0.05)$ of induction of apoptosis at $46 \mu M$ (Jurkat), resp. $32 \mu M(M O L M 13)$. Experiments were performed in triplicates. Non-linear regression analysis was performed to compute $I_{50 \text { s }}(\mathbf{b}$, e). Flow cytometry raw data are shown for Jurkat (c) and MOLM13 (f) cells demonstrating overwhelming induction of apoptosis in the highest tested doses - with no effect for methanol as drug carrier at the highest tested dose

using a flow cytometry-based assay. Antibody-specificity was validated by Western immunoblots and flow cytometry analysis using MOLM and Jurkat cell lines (Fig. 4a-b).

Marked CB1 as well as CB2 expression was confirmed in $4 / 12$ evaluated patients. Interestingly, expression of CB1 as well as CB2 was individually but equally elevated in these patients. The remaining 8 patients showed significantly lower expression levels of either of the receptors (Fig. 4c). Comparative evaluation of $\mathrm{CB}$ receptor expression levels in 10 healthy bone marrow donors revealed similar low expression levels.

Notably, correlation of CB-expression levels with responders to THC (defined as an apoptosis rate of at least $20 \%$ upon treatment with THC for $48 \mathrm{~h}$ ) revealed that expression of the cannabinoid receptors is a definite prerequisite to achieve any proapoptotic effect in native leukemia blasts.

\section{The proapoptotic effect of THC is mediated via CB1 - as well as CB2}

As both receptors were equally increased or diminished in all tested cell lines and patient samples, we asked whether the observed proapoptotic effect can be linked to one specific receptor.

We established an assay to specifically block the CB1 or CB2 receptor prior to exposure of leukemia cells to THC and used MOLM13 or Jurkat cells as a myeloid, respective lymphoid leukemia model:

LY320135, a highly selective cannabinoid receptor antagonist with a 70-fold higher affinity to CB1 than CB2 and a selective CB2 inverse ligand agonist (JTE-907) were first tested in dose-dependent dilution series in both cell lines to determine the optimal concentration without an intrinsic cell toxic effect (Fig. 5a).

Jurkat or MOLM13 cells were next treated with subtoxic doses of either LY320135 or JTE-907 at $0,1 \mu \mathrm{g} / \mathrm{ml}$ for $12 \mathrm{~h}$. THC was then administered at $\sim \mathrm{IC}_{50}$ doses and cells were incubated for an additional $48 \mathrm{~h}$. Notably, both inhibitors were able to abrogate THC-mediated induction of apoptosis in Jurkat cells as well as the MOLM13 cell line (Fig. 5b). As statistical analysis closely failed significance for CB2-interfered cell strains, we set up an alternative approach to confirm CB1- as well as CB2-dependency of the proapoptotic effect in leukemia cells: A knockout transfection approach was established using a CRISPR double nickase plasmid selectively encoding for CB1 or CB2. Puromycin selection was used to create stable CB1, resp. CB2 knockout cell strains of the Jurkat leukemia cell line. Importantly, knockdown of CB1 as well as CB2 resulted in highly significant abrogation of proapoptotic effects upon treatment with THC (see Additional file 9: Figure S9), supporting the finding of a direct role of either of the cannabinoid receptors in induction of apoptosis in acute leukemia models.

To confirm rescue from induction of apoptosis on the protein level, cleavage of caspase 3 (as an indicator of activated apoptosis signal transduction pathways) was determined by western immunoblot experiments. Indeed, THC-treated MOLM13 as well as Jurkat cells were

Table 1 Sensitivity of leukemia cell lines in response to THC

\begin{tabular}{|c|c|c|c|c|c|}
\hline \multirow{3}{*}{$\begin{array}{l}\text { Patient No. } \\
\text { Entitiy }\end{array}$} & \multirow{2}{*}{\multicolumn{3}{|c|}{$\begin{array}{l}\text { Phenotype } \\
\text { lineage dependency is marked (+) aberrantly expressed antigens are separately indicated }\end{array}$}} & \multicolumn{2}{|l|}{ THC response } \\
\hline & & & & \multirow{2}{*}{$\begin{array}{l}\text { \% viable } \\
\text { cells at } 50 \mu \mathrm{M}\end{array}$} & \multirow[t]{2}{*}{ IC50 ( $\mu \mathrm{M}$} \\
\hline & T-lymphatic & B-lymphatic & myeloid & & \\
\hline K562 & - & - & + & 87 & 62 \\
\hline M07e & - & - & + & 81 & 59 \\
\hline HL60 & CD4 & - & + & 15 & 38 \\
\hline Kasumi1 & CD4 & - & + & 14 & 35 \\
\hline MV4-11 & CD4 & - & + & 3 & 39 \\
\hline MOLM14 & CD4 & - & + & 18 & 44 \\
\hline MOLM13 & CD4 & - & + & 3 & 33 \\
\hline Jurkat & + & - & - & 31 & 46 \\
\hline
\end{tabular}




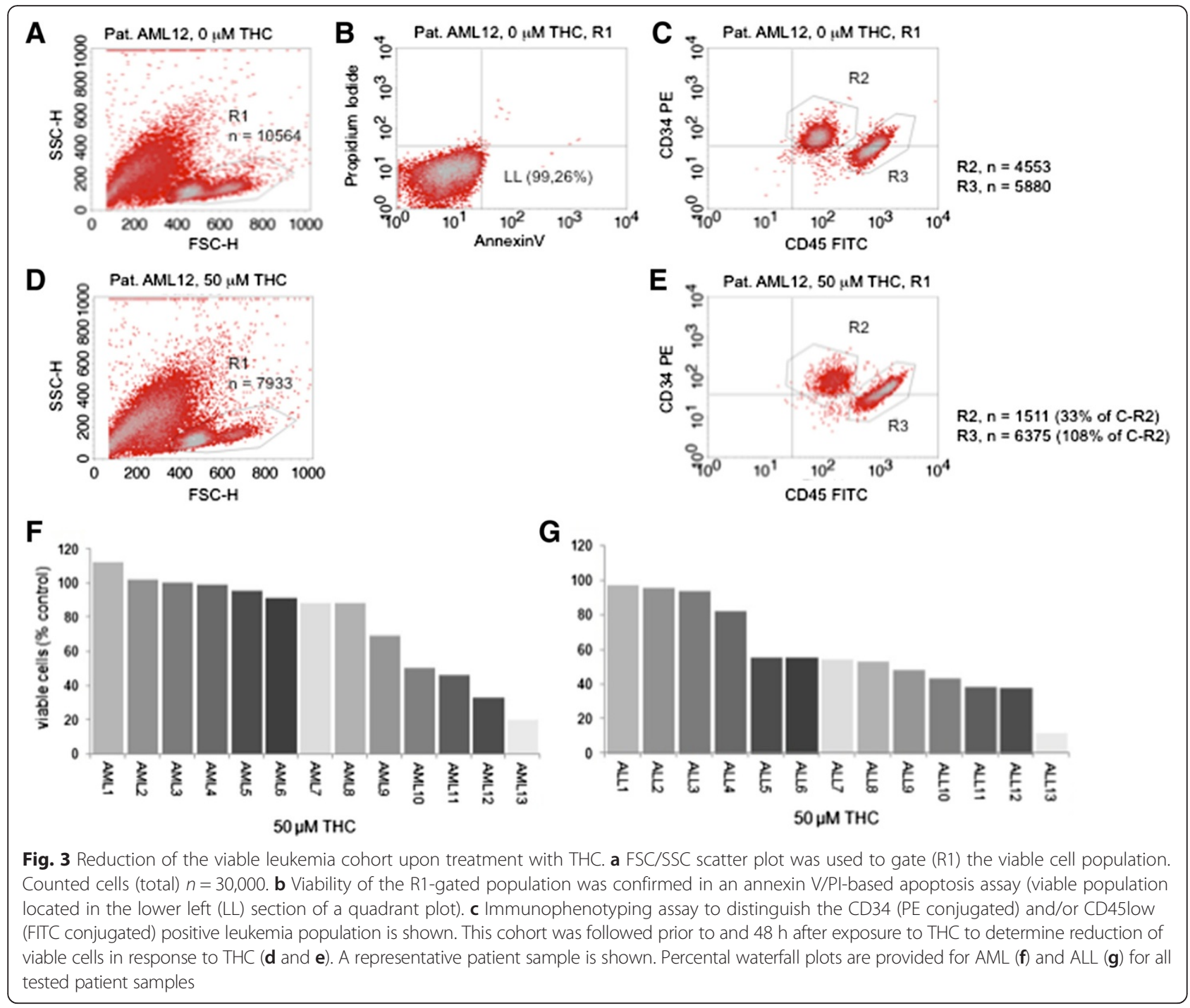

successfully rescued from caspase 3 cleavage after pretreatment of cells with LY320135 or JTE-907 (Fig. 5c).

\section{Response to THC is higher in leukemia blasts expressing lymphatic markers}

As demonstrated in Fig. 3, responses to THC were predominantly seen in acute leukemia entities derived from the lymphatic lineage. However, there was a subset of myeloid leukemia patient samples that had considerable sensitivity towards THC as well.

In an attempt, to further define the cohort responsive towards THC, we performed a systematic review of all available expression markers obtained at diagnosis-and found that most sensitive AML samples aberrantly expressed (T-) lymphoid differentiation markers (Table 2).

In this context, it is utmost remarkable, that all analyzed leukemia cell lines with higher sensitivity towards
THC aberrantly express T-lymphatic antigens as well (summarized in Table 1, see DSMZ homepage and Matsuo et al. [19] for expression profiles of cell lines).

However, as ALL samples with sensitivity towards THC were not restricted to the T-lineage, the observation of linking T-cell markers with THC-response may be biased due to the limited number of samples analyzed - and AML cohorts expressing B-cell markers may respond to THC as well. In our tested cohort, the only case expressing a B-differentiation marker (CD19, AML-6) did not show significant sensitivity towards THC up to $50 \mu \mathrm{M}$.

\section{Discussion}

Treatment outcome for acute leukemia in adults is still unsatisfactory for most entities. Besides disease-specific limitations such as high-risk genomic or chromosomal aberrations, comorbidities need to be addressed, especially in the increasing elderly population, restricting 


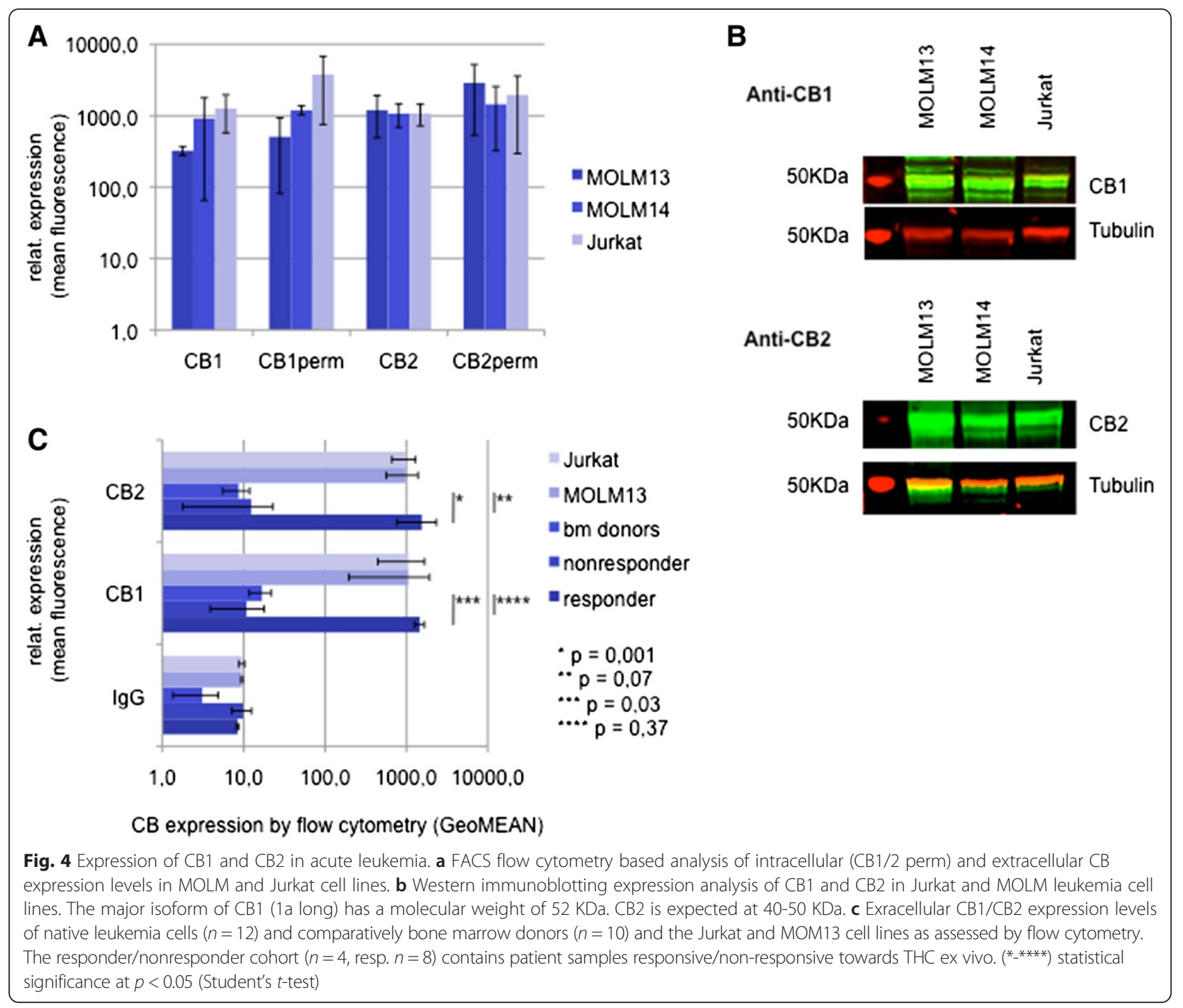

therapeutic options to epigenetic approaches, symptomatic cytoreduction or best supportive care.

We here reveal a novel aspect of dronabinol, a cannabinoid derivative, which displays remarkable antiproliferative as well as proapoptotic efficacy in a distinct leukemia patient cohort - in vitro and in ex vivo native leukemia blasts. It has been previously reported that cannabinoids display anticancer properties. However, due to legal issues the use and exploration of such agents is highly limited in many countries. Definition of dosing and entities benefitting from these agents remain vague and despite mounting evidence regarding their anti-tumorous effects cannabinoids have not been further developed as anticancer agents.

Even more challenging, controversial data suggest that cannabinoid agonists may foster tumorigenesis in some entities: For an acute myeloid leukemia model it has been demonstrated that CB2 has oncogene properties abrogating myeloid differentiation $[13,20]$.
We now provide rigorous proof-of-principle data demonstrating that (A) dronabinol has antiproliferative as well as proapoptotic efficacy in a broad spectrum of acute leukemia cell lines and native blasts cultured ex vivo and (B) this effect was preferentially observed in blasts with lymphoid differentiation or myeloid blasts aberrantly expressing lymphatic antigens. (C) The proapoptotic effect of dronabinol is mediated via $\mathrm{CB} 1$ as well as CB2 - and expression of the CB receptors is a prerequisite for therapy response. (D) Antitumor efficacy is dose-dependent and achievable in vivo.

Despite numerous reports on the anti-cancerous efficacy of THC the mechanisms of action as well as defined responder populations still remain unclear. Our data demonstrating antiproliferative as well as proapoptotic efficacy in defined acute leukemia models as well as ex vivo patient samples thereby aims to define a patient sample cohort potentially profiting from dronabinol 


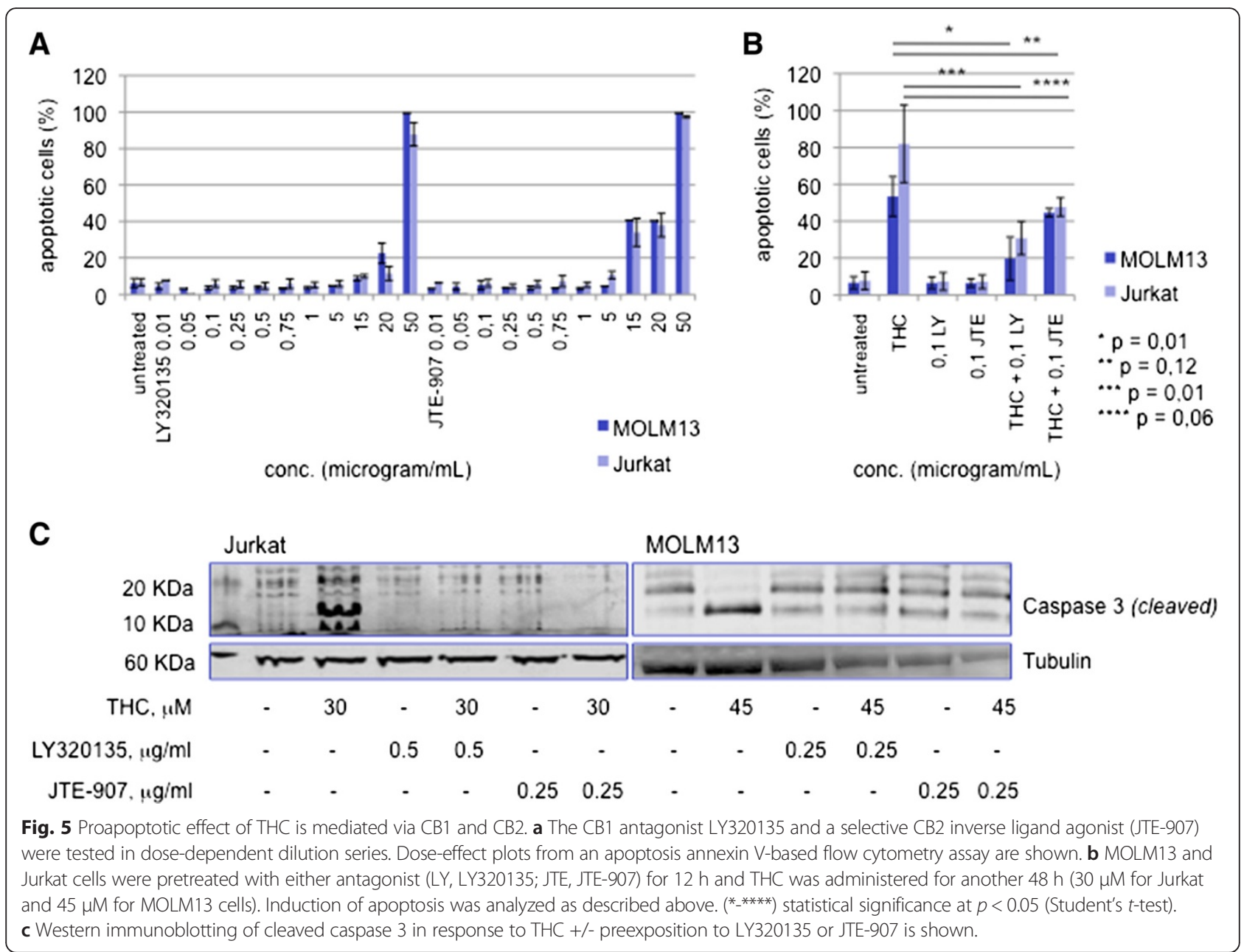

therapy. The observation that lymphoid blasts or myeloid samples expressing lymphatic markers are more sensitive towards THC is extremely valuable for therapeutic decisions and the observed lineage-dependency might explain the controversial results observed for cannabinoid activation in acute leukemia models in the past. But studies on a larger patient cohort are necessary to verify our observation and future studies will have to address the underlying mechanisms.

The mediation via both cannabinoid receptors CB1 and CB2 was verified using two different strategies - by transient silencing receptor activity via specific antagonists and by CRISPR double nickase knockdown. Our in vitro data is thereby backed up by the observation that all patient samples sensitive towards THC presented with high protein expression levels of $\mathrm{CB} 1$ and $\mathrm{CB} 2$ receptors whereas vice versa all non-responder displayed only low $\mathrm{CB} 1 / 2 \mathrm{ex}-$ pression. We thus believe to shed new light into the identification of a potential responder cohort. Importantly, we show that the healthy bone marrow donor population displays comparatively low CB1/2 expression as well. This is important to assess and evaluate the necessary doses and potential side effects.

Due to the excellent safety profile of dronabinol (compare drug information of $\mathrm{Marinol}^{\circ}$ ) effective doses are achievable in vivo. However, individual tolerable doses may vary widely-and starting with a sub-effective dose to be increased gradually may be necessary to build up tolerance to the well known psychoactive effects.

In this context, we had the opportunity to extract plasma from an elderly patient treated with dronabinol under palliative supportive care considerations for tumor kachexia. Dronabinol, provided by the university hospital's pharmacy as $2.5 \%$ oily solution, was started with 2 drops bid and tampered to 6 drops bid without any side effects. The patient was not treated with any antitumor or cytoreductive therapy. Plasma was used to culture Jurkat cells-and a considerable plasma inhibitory effect was documented in an apoptosis assay (Additional file 10: Figure S10). This observation argues for an antileukemic activity of dronabinol in vivo. 
Table 2 Sensitivity of native leukemia blasts in response to THC

\begin{tabular}{|c|c|c|c|c|}
\hline \multirow{3}{*}{$\begin{array}{l}\text { Patient No. } \\
\text { Entity }\end{array}$} & \multirow{2}{*}{\multicolumn{3}{|c|}{$\begin{array}{l}\text { Phenotype } \\
\text { lineage dependency is marked (+) } \\
\text { aberrantly expressed antigens are } \\
\text { separately indicated }\end{array}$}} & \multirow{3}{*}{$\begin{array}{l}\text { THC response } \\
\% \text { viable cells } \\
\text { at } 50 \mu \mathrm{M}\end{array}$} \\
\hline & & & & \\
\hline & T-lymphatic & B-lymphatic & myeloid & \\
\hline \multicolumn{5}{|l|}{ AML-1 } \\
\hline SAML (MDS) & N/A & N/A & + & 100 \\
\hline \multicolumn{5}{|l|}{ AML-2 } \\
\hline AML, FLT3-ITD & - & - & + & 100 \\
\hline \multicolumn{5}{|l|}{ AML-3 } \\
\hline AML NOS & (CD3) & - & + & 100 \\
\hline \multicolumn{5}{|l|}{$A M L-4$} \\
\hline$A P L$ & - & - & + & 99 \\
\hline \multicolumn{5}{|l|}{ AML-5 } \\
\hline AML, FLT3-ITD & - & - & + & 96 \\
\hline \multicolumn{5}{|l|}{ AML-6 } \\
\hline AML, FLT3-ITD & - & CD19 & + & 91 \\
\hline \multicolumn{5}{|l|}{ AML-7 } \\
\hline AML NOS (MO) & - & - & + & 89 \\
\hline \multicolumn{5}{|l|}{ AML-8 } \\
\hline AML, FLT3-ITD & - & - & + & 88 \\
\hline \multicolumn{5}{|l|}{ AML-9 } \\
\hline$N / A$ & N/A & N/A & + & 69 \\
\hline AML-10 & CD7 & & & \\
\hline AML, FLT3-ITD & CD56 & - & + & 50 \\
\hline AML-11 & CD7 & & & \\
\hline SAML (MDS) & CD5 & - & + & 46 \\
\hline AML-12 & $(\mathrm{CD} 7)$ & & & \\
\hline AML NOS (MO) & (CD5) & - & + & 33 \\
\hline AML-13 & CD7 & & & \\
\hline CBF AML & CD5 & - & + & 20 \\
\hline \multicolumn{5}{|l|}{ ALL-1 } \\
\hline (c) B-ALL & - & + & - & 97 \\
\hline ALL-2 & & & CD33 & \\
\hline pre B-ALL & - & + & CD13 & 96 \\
\hline \multicolumn{5}{|l|}{ ALL-3 } \\
\hline (c) B-ALL & - & + & CD33 & 94 \\
\hline \multicolumn{5}{|l|}{ ALL-4 } \\
\hline pre-B-ALL & - & + & - & 82 \\
\hline \multicolumn{5}{|l|}{ ALL-5 } \\
\hline (c) B-ALL & - & + & - & 55 \\
\hline \multicolumn{5}{|l|}{ ALL-6 } \\
\hline Cortical T-ALL & + & CD79 & - & 55 \\
\hline \multicolumn{5}{|l|}{ ALL-7 } \\
\hline (c) B-ALL & - & + & - & 54 \\
\hline ALL-8 & & + & CD33 & \\
\hline
\end{tabular}

Table 2 Sensitivity of native leukemia blasts in response to THC (Continued)

\begin{tabular}{lllll}
\hline pre $B$-ALL & - & CD10 & CD13 & 53 \\
ALL-9 & & & & \\
(c) B-ALL & - & + & - & 48 \\
ALL-10 & & & & \\
(c) B-ALL & - & + & - & 43 \\
ALL-11 & & & & \\
Cortical T-ALL & + & - & - & 38 \\
ALL-12 & & & CD33 & \\
(c) B-ALL & - & + & CD13 & 37 \\
ALL-13 & CD56 & & & \\
pre B-ALL & CD1a & + & CD13 & 12 \\
\hline
\end{tabular}

Due to sparse densities of cannabinoid receptors in lower brainstem areas, which control cardiovascular and respiratory functions, severe intoxications with $\mathrm{THC}$ have rarely been reported [21]. $\mathrm{LC}_{50 \mathrm{~s}}$ are not well defined (lethal concentration for male rats were $1270 \mathrm{mg} / \mathrm{kg}$ when orally administered; compare http://toxnet.nlm.nih.gov) and doselimiting side effects may be due to cardiovascular effects by lowering blood pressure and heart rate [22]. In this context it is also important to mention that healthy tissues tend to exhibit lower densities of cannabinoid receptors compared to malignant tissues (see expression data described herein or e.g. Kerner et al. who report on significantly higher CB2 expression in glioblastoma in comparison to healthy brain tissue). These findings suggest that therapeutically relevant and at the same time well tolerated proapoptotic doses can be achieved in acute leukemias.

Importantly, our data is in line with findings of others that have reported on the proapoptotic effect of cannabinoids in leukemia cell lines [22, 23]. Discrepancies for $\mathrm{IC}_{50 \mathrm{~s}}$ of $\mathrm{THC}$ derivatives reported within different studies are likely due to the known instability and origin of the compound, differences in the chosen time intervals between treatment and analyses, and differing cell culture conditions, including FBS concentrations. In this context, we have previously shown that FBS conditions may have significant impact on in vitro sensitivity profiles of tumor cells towards chemo- or targeted therapeutics, linked to direct drug-protein interactions and indirectly via effects on cell cycle regulation [17, 24]. Thus, our data provides a proof-of-principle, but effective clinical doses will need to be determined in vivo.

Cannabinoid receptor agonists as low-toxic agents may be especially of interest in the context of heavily pretreated, elderly or therapy refractory disease. Notably, we have evidence that dronabinol retained antileukemic activity in a sample of an otherwise chemotherapy and steroid-refractory ALL patient (see Additional file 11: Figure S11). 
In this context, a case report of a 14 year old girl with refractory $B C R-A B L 1(\mathrm{Ph}+)$ ALL was recently published demonstrating dramatic blast reduction in an individual therapy approach using escalating doses of a cannabis extract [25]. It is remarkable, that the selected case fits into the defined responder cohort of our study.

The compiled data demonstrates impressively, that dronabinol should be considered in selected cases of patients with acute leukemia but also stresses on the importance of thoroughly reflecting on the individual expression profiles of $\mathrm{CB} 1 / \mathrm{CB} 2$ as well as on additional diagnostic criteria-as e.g. lymphatic markers.

Even though it is not the intended purpose of this article, it should not stay unmentioned that besides the direct anti-leukemic effects of dronabinol the therapeutical use of $\mathrm{THC}$ in this patient cohort might exhibit a multitude of positive, desirable side effects like general physical well-being, cachexia control as well as pain, anxiety and stress relief, and thus should facilitate the decision process.

\section{Conclusion}

To summarize, we provide a promising rationale for the clinical use of cannabinoids, such as dronabinol, in distinct entities of acute leukemia-and this approach should further be evaluated.

\section{Methods \\ Cell lines}

The CML blast crisis cell line K562, the MLL-AF9 fusion positive acute myelogenous leukemia cell lines MOLM13 and the sister cell line MOLM14, both deriving from the same patient [26], and the human hematopoietic growth factor-dependent M-07e cell line were kindly provided by Drs. Heinrich and Lopez, Oregon Health and Science University, Portland, OR. The acute T-cell lymphoblastic leukemia cell line Jurkat, the AML cell lines HL60 and MV4-11 and the core binding factor leukemia cell line Kasumi1 [27] were obtained from the German Collection of Microorganisms and Cell Cultures (DSMZ).

Cells were cultured in RPMI 1640, supplemented with $10 \%$ fetal bovine serum, $1 \%$ penicillin G (10,000 units/ $\mathrm{mL})$, and streptomycin $(10,000 \mu \mathrm{g} / \mathrm{mg})(\mathrm{GIBCO} /$ Invitrogen, Darmstadt, Germany or BiochromAG, Berlin, Germany). Negativity for mycoplasma contamination was confirmed using the pluripotent PCR Mycoplasma test kit (AppliChem, Darmstadt, Germany). Cell lines harboring a mutant KIT (Kasumi1), FLT3 (MOLM13; MOLM14, MV4-11) or ABL (K562) isoform were sequence confirmed. M-07e cells were cultured using $10 \mathrm{ng} / \mathrm{ml}$ recombinant human granulocyte-macrophage colony stimulating factor (GM-CSF) as a growth supplement.

\section{Reagents}

Dronabinol (i.e. (-)- $\Delta^{9}$-Tetrahydrocannabinol, THC), dissolved in methanol, was obtained from THC Pharm (Frankfurt/Main, Germany) with permission of the Federal Opium Agency at the Federal Institute for Drugs and Medical Device, Germany. The selective CB1 antagonist LY320135 and the selective CB2 inverse agonist JTE-907 (CB2) were purchased from Sigma (St. Louis, MO).

\section{Isolation of bone marrow and peripheral blood mononuclear cells}

Bone marrow aspirate and peripheral blood samples from patients with diagnosed acute leukemia were collected in $5000 \mathrm{U}$ heparin after written informed consent, including publication of the data, and approval of the ethics committee of the University of Tübingen. Mononuclear cells were isolated by Ficoll Hypaque density gradient fractionation [17].

\section{Immunoblotting}

Cell pellets were lysed with 100 to $150 \mu \mathrm{L}$ of protein lysis buffer $(50 \mathrm{mmol} / \mathrm{L}$ Tris, $150 \mathrm{mmol} / \mathrm{L} \mathrm{NaCl}, 1 \%$ NP40, $0.25 \%$ deoxycholate with added inhibitors aprotinin, AEBSF, leupeptin, pepstatin, sodium orthovanadate, and sodium pyruvate, respectively phosphatase inhibitor cocktails „2“and „1“or „3“(Sigma, St. Louis, MO). Protein from cell lysates (75 to $200 \mu \mathrm{g}$ protein) was used for whole cell protein analysis after denaturing by Western immunoblot assays using a BioRad Criterion system (protein separation by SDS-PAGE in 3-8 \% or $10 \%$ polyacrylamide gels followed by electroblotting onto nitrocellulose membranes). Nonspecific binding was blocked by incubating the blots in nonfat dry milk or BSA. Primary antibodies were incubated for one hour or over night, followed by several washes of Tris-buffered saline (TBS) containing $0.005 \%$ Tween 20. Goat antihuman cannabinoid receptor 1 or 2 (CB1/CB2) antibodies were purchased from Sigma (St. Louis, MO); rabbit anti-human cleaved caspase 3 as well as 9 and rabbit anti-mouse tubulin antibodies were obtained from Cell Signaling Technology (Danvers, MA). The major isoform of CB1 (1a long) has a molecular weight of 52 $\mathrm{KDa}$. The molecular weight of $\mathrm{CB} 2$ is $39 \mathrm{KDa}$ - and the corresponding band in the immunoblot for the used antibody is expected at $40-50 \mathrm{KDa}$ according to the manufacturer's protocol. Donkey anti-goat/rabbit/mouse infrared dye-conjugated secondary antibodies for the LI$\mathrm{COR}^{\circ}$ imaging detection system were used according to standard protocols (LI-COR Biosciences, Lincoln, NE). Secondary antibodies were applicated for 30, followed by several washes. Antibody-reactive proteins were detected using a LI-COR Odyssey ${ }^{\circ}$ fluorescence optical system (LI-COR Biosciences, Lincoln, NE) [17]. 


\section{Apoptosis assays}

Translocation of phosphatidylserine from the inner to the outer leaflet of the plasma membrane as an early indicator of apoptosis was analyzed using an annexin V-based assay (Immunotech, Marseilles, France) and a FACScalibur ${ }^{\circ}$ flow cytometer loaded with CellQuest ${ }^{\circ}$ analysis software (BD, Heidelberg, Germany) [28].

\section{Proliferation assays}

Cellular proliferation capacity was measured using an 2,3-bis[2-methoxy-4-nitro-5-sulfophenyl]-2H-tetrazolium5-carboxanilide inner salt (XTT)-based assay (Sigma, MO) [28].

\section{Immunophenotyping}

A routine panel for newly diagnosed acute leukemia was performed for every patient following standard in-house protocols. In addition, rabbit anti-human CB1 or CB2 antibodies (Cell Signaling Technology, Danvers, MA) were conjugated with fluorescent polyclonal secondary anti-rabbit IgG-H\&L (FITC) antibodies (Cell Signaling Technology as well) according to the manufacturer protocol and protein expression levels were assessed by flow cytometry using standard protocols.

\section{Data analysis}

Dose-effect plots were created to calculate $\mathrm{IC}_{50 \text { s }}$ using Prism 5.0 Software available from Graph Pad, La Jolla, CA.

\section{Additional files}

Additional file 1: Figure S1. Induction of apoptosis determined by externalization of phosphatidylserine. Jurkat cells are treated with THC for $10 \mathrm{~h}$ and analyzed using a flow cytometry annexin $\mathrm{V}$ staining protocol. Histograms of representative experiments are provided. (TIFF $432 \mathrm{~kb}$ )

Additional file 2: Figure S2. Proapoptotic effect of $\mathrm{THC}$ is mediated via the mitochondrial intrinsic pathway. Western immunoblotting of cleaved caspase 9 in Jurkat cells treated with THC is shown. Tubulin serves as a loading control. (TIFF 107 kb)

Additional file 3: Figure S3. Flow cytometric apoptosis assay. Dose-effect curves for leukemia cell lines treated with THC in a dose-dependent manner are shown. Student's t-test analysis demonstrates significance $(p<0.05)$ of induction of apoptosis. Experiments were performed in triplicates. Methanol as drug carrier was applicated at the highest tested dose (left panels). Non-linear regression analysis was performed to compute $\mathrm{IC}_{50}$ (right panels). (TIFF $557 \mathrm{~kb}$ )

Additional file 4: Figure S4. Flow cytometric apoptosis assay. Dose-effect curves for leukemia cell lines treated with THC in a dose-dependent manner are shown. Student's t-test analysis demonstrates significance $(p<0.05)$ of induction of apoptosis. Experiments were performed in triplicates. Methanol as drug carrier was applicated at the highest tested dose (left panels). Non-linear regression analysis was performed to compute $I_{50}$ (right panels). (TIFF $579 \mathrm{~kb}$ )

Additional file 5: Figure S5. Flow cytometric apoptosis assay. Dose-effect curves for leukemia cell lines treated with THC in a dose-dependent manner are shown. Student's $t$-test analysis demonstrates significance $(p<0.05)$ of induction of apoptosis. Experiments were performed in triplicates. Methanol as drug carrier was applicated at the highest tested dose (left panels). Non-linear regression analysis was performed to compute IC50s (right panels). (TIFF $567 \mathrm{~kb}$ )

Additional file 6: Figure S6. Flow cytometric apoptosis assay. Dose-effect curves for leukemia cell lines treated with THC in a dose-dependent manner are shown. Student's t-test analysis demonstrates significance $(p<0.05)$ of induction of apoptosis. Experiments were performed in triplicates. Methanol as drug carrier was applicated at the highest tested dose (left panels). Non-linear regression analysis was performed to compute IC50s (right panels). (TIFF $536 \mathrm{~kb}$ )

Additional file 7: Figure S7. Flow cytometric apoptosis assay. Dose-effect curves for leukemia cell lines treated with THC in a dose-dependent manner are shown. Student's $t$-test analysis demonstrates significance $(p<0.05)$ of induction of apoptosis. Experiments were performed in triplicates. Methanol as drug carrier was applicated at the highest tested dose (left panels). Non-linear regression analysis was performed to compute IC50s (right panels). (TIFF 527 kb)

Additional file 8: Figure S8. Flow cytometric apoptosis assay. Dose-effect curves for leukemia cell lines treated with $\mathrm{THC}$ in a dose-dependent manner are shown. Student's t-test analysis demonstrates significance $(p<0.05)$ of induction of apoptosis. Experiments were performed in triplicates. Methanol as drug carrier was applicated at the highest tested dose (left panels). Non-linear regression analysis was performed to compute IC50s (right panels). (TIFF $563 \mathrm{~kb}$ )

Additional file 9: Figure S9. Plasma inhibitory efficacy. Plasma derived from a patient supportively treated with dronabinol $\left(6^{\circ}\right.$ bid of a $2.5 \%$ oily solution) for tumor kachexia in a palliative setting was extracted and used to culture Jurkat leukemia cells for 48 and 72 h. Plasma inhibitory efficacy was analyzed in an annexin V/PI-based apoptosis assay. (TIFF 1292 kb)

Additional file 10: Figure S10. Reduction of the viable leukemia population upon treatment with THC. Immunphenotyping of the leukemic clone in a FSC/SSC scatter plot was performed in a patient with refractory $\mathrm{ALL}$ and $>90 \%$ blasts in the peripheral blood. Reduction of the population was followed after exposure to THC for $48 \mathrm{~h}$. Proportion of the remaining viable cell proportion is shown in a dose-effect plot. (TIFF $282 \mathrm{~kb}$ )

Additional file 11: Figure S11. Sensitivity of Jurkat leukemia cells towards THC after selective CB1-, resp. CB2, CRISPR knockdown. (A) Cells are transfected using standard protocols of the manufacturer (Santa Cruz) using a selective $C B 1$, respectively $C B 2, C R I S P R$ Double Nickase plasmid. GFP transfection efficiency control by flow cytometry after puromycin selection is shown. EV, empty vector negative control. (B) Validation of CRISPR knockdown of CB1, resp. CB2 protein expression using a flow cytometry approach. (C) Sensitivity of Jurkat cells towards THC (40 $\mu \mathrm{M})$ after selective $C B 1$, resp. CB2, interference (CB1i/CB2i) with regard to induction of apoptosis. Mean data of 3-5 independent annexin V/PIbased experiments are provided. $\left(^{*}{ }^{* *}\right)$ statistical significance at $p<0.05$ (Student's $t$-test). EV, empty vector. (TIFF $237 \mathrm{~kb}$ )

\section{Abbreviations}

ABL1: Abelson murine leukemia viral oncogene homolog 1; AML: acute myeloid leukemia; ALL: acute lymphoid leukemia; BSA: bovine serum albumin; CB1: cannabinoid receptor 1; CB2: cannabinoid receptor 2; CBFL: core binding factor leukemia; CML: chronic myeloid leukemia; DSMZ: Leibniz Institute, German Collection of Microorganisms and Cell Cultures; FACS: fluorescence-activated cell sorting; FITC: fluorescein isothiocyanate; FLT3: FMS-like tyrosine kinase 3; FSC: forward scatter (distiguishes volume of cells); $\mathrm{IC}_{50}$ : concentration sufficient to achieve a $50 \%$ inhibition; IL3: interleukin 3; ITD: internal tandem duplication; KIT: v-kit HardyZuckerman 4 feline sarcoma viral oncogene homolog; LC $_{50}$ : lethal concentration killing $50 \%$ ot the cohort; SSC: side scatter (distinguishes granularity of cells and size/shape of nucleus); PE: R-Phycoerythrin; THC: Delta9-Tetrahydrocannabinol; XTT: 2,3-Bis-(2-methoxy-4-nitro-5sulfophenyl)-2H-tetrazolium-5-carboxanilid-sodium salt.

\section{Competing interests}

Kerstin Kampa-Schittenhelm no conflicts. Olaf Salitzky no conflicts. Figen Akmut no conflicts. Barbara Illing no conflicts. Lothar Kanz no conflicts. Helmut Salih no conflicts. Marcus Schittenhelm no conflicts. 


\section{Authors' contributions}

KKS designed the research study, performed the research, analysed the data, wrote the paper; OS performed the research, analysed the data; FA performed the research; BI performed the research; LK analysed the data, wrote the paper; HS analysed the data, wrote the paper; MS designed the research study, analysed the data, wrote the paper. All authors have read and approved the manuscript, and ensure that this is the case.

\section{Acknowledgements}

Grant support in part by the Deutsche Krebshilfe Foundation (KKS), the IZKF Program of the Medical Faculty Tübingen (MMS), the Carreras Scholarship Program (KKS), the Brigitte-Schlieben-Lange Program (KKS) and the Athene Program (KKS). We acknowledge support by Deutsche Forschungsgemeinschaft and Open Access Publishing Fund of University of Tübingen.

\section{Received: 14 April 2015 Accepted: 17 December 2015}

Published online: 16 January 2016

\section{References}

1. Matsuda LA, Lolait SJ, Brownstein MJ, Young AC, Bonner TI. Structure of a cannabinoid receptor and functional expression of the cloned cDNA. Nature. 1990:346:561-4.

2. Munro S, Thomas KL, Abu-Shaar M. Molecular characterization of a peripheral receptor for cannabinoids. Nature. 1993;365:61-5.

3. Galve-Roperh I, Sanchez C, Cortes ML, Gomez del Pulgar T, Izquierdo M, Guzman M. Anti-tumoral action of cannabinoids: involvement of sustained ceramide accumulation and extracellular signal-regulated kinase activation. Nat Med. 2000;6:313-9.

4. Golech SA, McCarron RM, Chen Y, Bembry J, Lenz F, Mechoulam R, et al. Human brain endothelium: coexpression and function of vanilloid and endocannabinoid receptors. Brain Res Mol Brain Res. 2004;132:87-92.

5. Ofek O, Karsak M, Leclerc N, Fogel M, Frenkel B, Wright $K$, et al. Peripheral cannabinoid receptor, CB2, regulates bone mass. Proc Natl Acad Sci U S A. 2006;103:696-701

6. Casanova ML, Blazquez C, Martinez-Palacio J, Villanueva C, FernandezAcenero MJ, Huffman JW, et al. Inhibition of skin tumor growth and angiogenesis in vivo by activation of cannabinoid receptors. J Clin Invest. 2003; 111:43-50

7. Basu S, Dittel BN. Unraveling the complexities of cannabinoid receptor 2 (CB2) immune regulation in health and disease. Immunol Res. 2011;51:26-38.

8. Pertwee RG. Targeting the endocannabinoid system with cannabinoid receptor agonists: pharmacological strategies and therapeutic possibilities. Philos Trans R Soc Lond B Biol Sci. 2012;367:3353-63.

9. Guzman M. Cannabinoids: potential anticancer agents. Nat Rev Cancer. 2003:3:745-55

10. Lombard C, Nagarkatti M, Nagarkatti PS. Targeting cannabinoid receptors to treat leukemia: role of cross-talk between extrinsic and intrinsic pathways in Delta9-tetrahydrocannabinol (THC)-induced apoptosis of Jurkat cells. Leuk Res. 2005;29:915-22

11. Herrera B, Carracedo A, Diez-Zaera M, Guzman M, Velasco G. p38 MAPK is involved in CB2 receptor-induced apoptosis of human leukaemia cells. FEBS Lett. 2005;579:5084-8.

12. Sancho R, de la Vega L, Appendino G, Di Marzo V, Macho A, Munoz E. The CB1/NR1 agonist arvanil induces apoptosis through an FADD/caspase-8dependent pathway. Br J Pharmacol. 2003;140:1035-44.

13. Alberich Jorda M, Rayman N, Tas M, Verbakel SE, Battista N, van Lom K, et al. The peripheral cannabinoid receptor $\mathrm{Cb} 2$, frequently expressed on AML blasts, either induces a neutrophilic differentiation block or confers abnormal migration properties in a ligand-dependent manner. Blood. 2004;104:526-34.

14. Valk PJ, Delwel R. The peripheral cannabinoid receptor, Cb2, in retrovirallyinduced leukemic transformation and normal hematopoiesis. Leuk Lymphoma. 1998:32:29-43.

15. Valk P, Verbakel S, Vankan Y, Hol S, Mancham S, Ploemacher R, et al. Anandamide, a natural ligand for the peripheral cannabinoid receptor is a novel synergistic growth factor for hematopoietic cells. Blood. 1997;90:1448-57.

16. Carracedo A, Lorente M, Egia A, Blazquez C, Garcia S, Giroux V, et al. The stress-regulated protein p8 mediates cannabinoid-induced apoptosis of tumor cells. Cancer Cell. 2006;9:301-12.

17. Kampa-Schittenhelm KM, Heinrich MC, Akmut F, Rasp KH, Illing B, Dohner H, et al. Cell cycle-dependent activity of the novel dual PI3K-MTORC1/2 inhibitor NVP-BGT226 in acute leukemia. Mol Cancer. 2013;12:46.
18. Kampa-Schittenhelm KM, Heinrich MC, Akmut F, Dohner H, Dohner K, Schittenhelm MM. Quizartinib (AC220) is a potent second generation class III tyrosine kinase inhibitor that displays a distinct inhibition profile against mutant-FLT3, -PDGFRA and -KIT isoforms. Mol Cancer. 2013:12:19.

19. Matsuo Y, MacLeod RA, Uphoff CC, Drexler HG, Nishizaki C, Katayama Y, et al. Two acute monocytic leukemia (AML-M5a) cell lines (MOLM-13 and MOLM-14) with interclonal phenotypic heterogeneity showing MLL-AF9 fusion resulting from an occult chromosome insertion, ins $(11 ; 9)(q 23$; p22p23). Leukemia. 1997;11:1469-77.

20. Jorda MA, Rayman N, Valk P, De Wee E, Delwel R. Identification, characterization, and function of a novel oncogene: the peripheral cannabinoid receptor Cb2. Ann N Y Acad Sci. 2003;996:10-6.

21. Herkenham M, Lynn AB, Little MD, Johnson MR, Melvin LS, de Costa BR, et al. Cannabinoid receptor localization in brain. Proc Natl Acad Sci U S A. 1990:87:1932-6.

22. McKallip RJ, Lombard C, Fisher M, Martin BR, Ryu S, Grant S, et al. Targeting CB2 cannabinoid receptors as a novel therapy to treat malignant lymphoblastic disease. Blood. 2002;100:627-34.

23. Powles T, te Poele R, Shamash J, Chaplin T, Propper D, Joel S, et al. Cannabis-induced cytotoxicity in leukemic cell lines: the role of the cannabinoid receptors and the MAPK pathway. Blood. 2005;105:1214-21.

24. Koch S, Mayer F, Honecker F, Schittenhelm M, Bokemeyer C. Efficacy of cytotoxic agents used in the treatment of testicular germ cell tumours under normoxic and hypoxic conditions in vitro. Br J Cancer. 2003;89:2133-9.

25. Singh Y, Bali C. Cannabis extract treatment for terminal acute lymphoblastic leukemia with a Philadelphia chromosome mutation. Case Rep Oncol. 2013; 6:585-92.

26. Yokota S, Kiyoi H, Nakao M, Iwai T, Misawa S, Okuda T, et al. Internal tandem duplication of the FLT3 gene is preferentially seen in acute myeloid leukemia and myelodysplastic syndrome among various hematological malignancies. A study on a large series of patients and cell lines. Leukemia. 1997;11:1605-9.

27. Beghini A, Magnani I, Ripamonti CB, Larizza L. Amplification of a novel C-Kit activating mutation Asn(822)-Lys in the Kasumi-1 cell line: a t(8;21)-Kit mutant model for acute myeloid leukemia. Hematol J. 2002;3:157-63.

28. Schittenhelm MM, Shiraga S, Schroeder A, Corbin AS, Griffith D, Lee FY, et al. Dasatinib (BMS-354825), a dual SRC/ABL kinase inhibitor, inhibits the kinase activity of wild-type, juxtamembrane, and activation loop mutant KIT isoforms associated with human malignancies. Cancer Res. 2006;66:473-81.

\section{Submit your next manuscript to BioMed Central and we will help you at every step:}

- We accept pre-submission inquiries

- Our selector tool helps you to find the most relevant journal

- We provide round the clock customer support

- Convenient online submission

- Thorough peer review

- Inclusion in PubMed and all major indexing services

- Maximum visibility for your research

Submit your manuscript at www.biomedcentral.com/submit
) Biomed Central 